\title{
Queimadura oral por ingestão acidental de ácido: Relato de caso clínico
}

\author{
Oral Burn by acidental acid ingestion: Case report \\ Quemadura oral por ingestión acidental de ácido: Reporte de caso
}

Mirela Caroline Silva

ORCID: https://orcid.org/0000-0002-9455-3807

São Paulo State University, Brazil

E-mail: mirela_carol12@hotmail.com

William Phillip Pereira da Silva

ORCID: https://orcid.org/0000-0003-4172-7217

São Paulo State University, Brazil

E-mail: william_phillip@ hotmail.com

Lara Cristina Cunha Cervantes

ORCID: https://orcid.org/0000-0003-4448-2702

São Paulo State University, Brazil

E-mail: laraccerv@gmail.com

Stefany Barbosa

ORCID: https://orcid.org/0000-0002-4190-7931

São Paulo State University, Brazil

E-mail: stefanybarbosa61 @gmail.com

João Matheus Fonseca e Santos

ORCID: https://orcid.org/0000-0002-2021-778X

São Paulo State University, Brazil

E-mail: jmfs15@hotmail.com

Barbara Ribeiro Rios

ORCID: https://orcid.org/0000-0002-5389-5536

São Paulo State University, Brazil

E-mail: barbarariios@outlook.com

Franscisley Ávila de Souza

ORCID: https://orcid.org/0000-0002-1427-071X

São Paulo State University, Brazil

E-mail: franscisley.avila@unesp.br

Osvaldo Magro Filho

ORCID: https://orcid.org/0000-0002-9821-2479

São Paulo State University, Brazil

E-mail: osvaldo.magro@unesp.br

Idelmo Rangel Garcia Junior

ORCID: https://orcid.org/0000-0001-8892-781X

São Paulo State University, Brazil

E-mail: irgcirurgia@gmail.com

Daniela Ponzoni

ORCID: https://orcid.org/0000-0001-5928-0914

São Paulo State University, Brazil

E-mail: daniela.ponzoni@unesp.br

Ana Paula Farnezi Bassi

ORCID: https://orcid.org/0000-0002-0031-4953

São Paulo State University, Brazil

E-mail: ana.bassi@unesp.br

Alessandra Marcondes Aranega

ORCID: https://orcid.org/0000-0001-5856-7972

São Paulo State University, Brazil

E-mail: alessandra.aranega @unesp.br

Leonardo Perez Faverani

ORCID: https://orcid.org/0000-0003-2249-3048

São Paulo State University, Brazil

E-mail: leonardo.faverani@unesp.br

\section{Resumo}

Introdução: Queimaduras da mucosa oral podem ser ocasionadas devido traumas térmicos, químicos ou elétricos, podendo causar uma lesão severa não somente na mucosa oral, mas sim em região esôfago e estômago, levando a uma necrose tecidual ou até uma perfuração das estruturas. Objetivo e relato de caso: O presente artigo tem como objetivo relatar um caso clínico de ingestão acidental de substância ácida e realizar uma revisão de literatura discutindo a 
importância do cirurgião dentista na equipe multidisciplinar de um serviço de emergência, atuando no diagnóstico e tratamento das lesões de queimaduras químicas. Conclusão: Algo indispensável para a restauração da função e recuperação adequadas desses pacientes.

Palavras-chave: Ingestão; Substâncias ácidas; Trauma químico; Queimadura oral.

\begin{abstract}
Introduction: Burns of the oral mucosa can be caused due to thermal, chemical or electrical trauma, which can cause a severe lesion not only in the oral mucosa, but also in the esophagus and stomach, leading to tissue necrosis or even perforation of the structures. Objective and case report: This article aim is report a clinical case of accidental ingestion of acidic substance and perform a literature review discussing the importance of the dental surgeon in the multidisciplinary team of an emergency service, acting in the diagnosis and treatment of injuries of chemical burns. Conclusion: Something indispensable for the restoration of proper function and recovery of these patients.
\end{abstract}

Keywords: Ingestion; Acid substances; Chemical trauma; Oral burns.

\title{
Resumen
}

Introducción: Las quemaduras de la mucosa oral pueden ser causadas por traumatismos térmicos, químicos o eléctricos, que pueden ocasionar una lesión grave no solo en la mucosa oral, sino también en el esófago y estómago, dando lugar a necrosis tisular o incluso perforación del estructuras. Objetivo y caso clínico: Este artículo tiene como objetivo reportar un caso clínico de ingestión accidental de sustancia ácida y realizar una revisión de la literatura discutiendo la importancia del cirujano dentista en el equipo multidisciplinario de un servicio de urgencias, actuando en el diagnóstico y tratamiento de lesiones de origen químico. quemaduras Conclusión: Algo indispensable para el restablecimiento de la función y la adecuada recuperación de estos pacientes.

Palabras clave: Ingéstion; Substancias ácidas; Trauma químico; Quemadura oral.

\section{Introdução}

Queimaduras da mucosa oral podem ser ocasionadas devido traumas térmicos, químicos ou elétricos, afetando principalmente os lábios, língua e palato (Alfawaz, 2020; Baruchin, Lustig, Nahlieli, \& Neder, 1991; S. Kang, Kufta, Sollecito, \& Panchal, 2017). A deglutição de substâncias químicas acidentalmente ou propositalmente como ácidos, cloro, detergentes, álcalis, podem causar uma lesão severa não somente na mucosa oral, mas também em região de esôfago e estômago, levando a uma necrose tecidual ou até uma perfuração das estruturas. O grau de comprometimento varia de acordo com o tipo da substância química, duração e extensão de contato com o corpo (Bahnof, 2000; Cowan, Ho, Sykes, \& Wei, 2013).

Ingestão acidental de agentes químicos, geralmente, produzem danos mais superficiais a mucosa oral devido ao sabor adverso e forte odor, o que resulta na regurgitação da substância, limitando os danos (Arévalo-Silva, Eliashar, Wohlgelernter, Elidan, \& Gross, 2006; Steve Kang, Kufta, Sollecito, \& Panchal, 2018; Pushp Chander Swami, Rushik Raval, Mandeep Kaur, \& Jasleen Kaur, 2016). A restrição de lesões apenas à mucosa torna o processo de cicatrização facilitado devido a características intrínsecas e locais do tecido, com formação quase nula de sequelas funcionais e/ou estéticas (Arévalo-Silva et al., 2006; Sundararajan, Noonan, \& Gallagher, 2017).

Independente da gravidade, o tratamento consiste no controle da sintomatologia, da infecção para que ocorra o reparo das feridas (Arévalo-Silva et al., 2006; Bahnof, 2000; Spranley, Winkler, Dagate, Oncale, \& Strother, 2012). Além disso o acompanhamento deve ser rigoroso para controlar possíveis extensões dos danos e evitar complicações (Pushp Chander Swami et al., 2016).

O presente artigo tem como objetivo relatar um caso clínico de ingestão acidental de substância ácida e realizar uma revisão de literatura discutindo a importância do cirurgião dentista na equipe multidisciplinar de um serviço de emergência, atuando no diagnóstico e tratamento das lesões de queimaduras químicas.

\section{Metodologia}

Trata-se de um estudo retrospectivo, descritivo e observacional no formato de Relato de Caso Clínico de um paciente tratado em ambiente hospitalar por uma equipe Qumultidisciplinar. Os dados epidemiológicos, história da doença e registros 
fotográficos foram coletados através do prontuário físico e eletrônico, após autorização dos responsáveis pelo paciente, mediante também a assinatura do Termo de Consentimento Livre e Esclarecido (TCLE), seguindo os preceitos para publicação sem a identificação do paciente.

\section{Relato de Caso}

Paciente do sexo masculino, 58 anos de idade, foi atendido no pronto socorro da Santa Casa de Misericórdia de Araçatuba pelo serviço de emergência, devido ingestão de substância ácida acidental. Paciente apresentava-se orientado, sem relato de perda de consciência e emese após a ingestão, não sabendo relatar qual substância foi ingerida e a quantidade. Em avaliação médica não foi diagnosticado alterações em esôfago e estômago, sendo encaminhado para a equipe de cirurgia bucomaxilofacial para avaliação da cavidade oral. Ao exame físico intra oral, paciente apresentava edema em lábios superior e inferior com lesão por queimadura grau 3 do lado esquerdo, com queixas álgicas intensas (Figura $1 \mathrm{~A}$ ). Como conduta foi realizado a orientação e prescrição de pomada anestésica e cicatrizante em orabase para alívio da sensação dolorosa, principalmente durante a alimentação e após a higienização oral. Foi realizado a prescrição de um medicamento protetor gástrico, analgésico e antibioticoterapia preventiva. Após 7 dias, o paciente retornou ao ambulatório para acompanhamento, o qual apresentava regressão total de edema e regressão total de queixas álgicas, recebendo alta da equipe (Figura 1 B-C).

Figura 1 - Lesão em toda extensão do lábio inferior (A); Aspecto da lesão após 7 dias que o tratamento instituído (B e C).

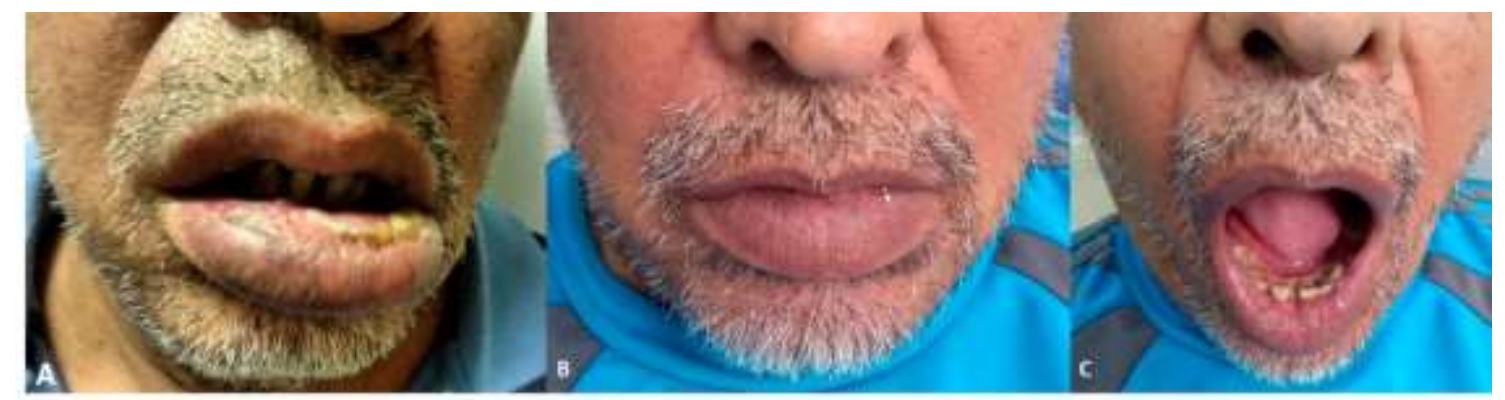

Fonte: Autores (2021).

\section{Discussão}

A gravidade das queimaduras químicas na mucosa oral, depende das propriedades destrutivas do agente cáustico, o modo, a duração e a extensão do seu contato com o corpo (Arevalo-Silva, Eliashar, Wohlgelernter, Elidan, \& Gross, 2006; Bahnof, 2000; Kurowski \& Kay, 2017; Rallan, Malhotra, Rallan, \& Mayall, 2013; Swami, Raval, Kaur, \& Kaur, 2016; Wason, 1985). Diferentemente de uma ingestão proposital a qual o paciente acaba engolindo uma grande quantidade da substância química, a ingestão acidental geralmente é expelida rapidamente, devido ao forte odor e sabor desagradável dos agentes cáusticos, causando assim um trauma menor aos tecidos. Em nosso caso clínico, o paciente não sabia relatar qual foi a substância química e a quantidade que ingeriu, porém no exame clínico, foi observado apenas lesões de queimadura em mucosa bucal, com ausência de comprometimento em esôfago e estomago, podendo concluir que a ingestão foi em pouca quantidade. Assim como relatado na literatura, a maioria dos agentes cáusticos frequentemente têm um forte odor e um sabor desagradável, sendo geralmente expelidos rapidamente, a não ser que a ingestão seja intencional (Alfawaz, 2020; Bahnof, 2000). A mucosa oral é composta de tecidos queratinizados (palato duro e gengiva inserida), epitélio escamoso estratificado não queratinizado, papilas epiteliais (dorso da língua), mucosa de revestimento (lábios, bochechas, vestíbulos, assoalho de 
boca e ventre da língua), tal como a pele, as funções da mucosa são para servir como barreira protetora, mecânica e microbiológica (Evans, 2017; Hagiwara, Seki, \& Takahashi, 2020).

Porém quando se compara a resposta diante de queimaduras, a mucosa tem pouca semelhança com a pele. Queimaduras em mucosas tendem a cicatrizar mais rapidamente com formação mínima ou nula de feridas, tendo um impacto quase inexistente em relação a função e a estética. Isso se dá pela rápida remodelação, relacionada às características intrínsecas existente apenas nos tecidos mucosos e a fatores ambientais, como a umidade e a microflora. Autores afirmam que esse tipo de cicatrização é considerada ideal por ocorrer mais rapidamente e devido ao tecido cicatricial sequer formar, na maioria dos casos (Erik William Evans, 2017; Haller Jr, Andrews, White, Tamer, \& Cleveland, 1971; Steve Kang et al., 2018; Nehrlich, Klöcking, Hentschel, \& Lupp, 2017). No presente caso, a queimadura ocorreu apenas em mucosa oral o que justifica a ausência de intercorrências quanto a cicatrização, a formação de cicatrizes e a melhora do quadro em apenas 7 dias.

A maioria dos danos ocorre na orofaringe, faringe, amigdalas, mucosa da língua, mucosa do palato ou gengiva e pode ser de forma mais localizada ou difusa, com um diagnóstico puramente clínico. A complicação mais grave é a necrose de estômago e/ou esôfago, o que pode levar a septicemia e até a morte. Isso denota a necessidade de acompanhamento constante do paciente, pois essas complicações podem ocorrer poucas horas após a ingestão; porém, mais comumente, após 72 horas a duas semanas após o incidente. Além disso, a presença ou ausência de lesões orofaríngeas não indica com segurança os danos reais que podem ter acontecido a tecidos mais profundos, por isso, há necessidade de um alerta vigoroso durante a terapia dos pacientes (Alfawaz, 2020; Clayton, Ward, \& Maitz, 2015; Lopes et al., 2020; Vargo, Warner, Potluri, \& Prasad, 2017). O paciente em questão passou por um acompanhamento extenso após o ocorrido, mesmo que não tenha sido diagnosticado com alterações no esôfago e estômago pela equipe médica.

$\mathrm{O}$ tratamento de queimaduras, independente da gravidade, tem por objetivos o controle da dor, da infecção e o reparo das feridas causadas. Para isso, o manejo do paciente deve ser compatível a história médica e com a natureza da lesão, levando em consideração a etiologia, duração e distribuição dos danos (Arévalo-Silva et al., 2006; Hagiwara et al., 2020). Especialmente em casos de queimaduras limitadas a mucosa oral, devido a suas características inerentes quanto a cicatrização; a terapia se dá de forma mais sintomática (Bahnof, 2000; Pushp Chander Swami et al., 2016). Antibióticos podem ser utilizados para prevenção de infecções tanto da mucoca oral quanto se houver lesões em tecidos mais profundos, mesmo que seu uso ainda seja controverso (Jacob A Kurowski \& Marsha Kay, 2017). Nesse caso, optou-se por um tratamento sintomático devido a limitação do dano a mucosa oral, a partir da prescrição de pomadas analgésicas e analgésico sistêmico e, como diligência, optou-se pelo uso de antibióticos e protetor gástrico.

De modo geral, prevenção é a forma mais efetiva de evitar acontecimentos, mesmo que acidentais, como o descrito. Isso se dá a partir da rotulação e do manuseio correto de agentes químicos que podem levar a queimaduras, além de orientações fornecidas em casos de acidentes para que o mesmo não tenha recorrência. E caso o mesmo ocorra, o profissional tem papel fundamental em atingir os objetivos do tratamento para que o paciente recupere o tecido acometido de maneira mais saudável possível a função e a estética (Arévalo-Silva et al., 2006; Bahnof, 2000; Edlich, Farinholt, Winters, Britt, \& Long III, 2005; Nichter et al., 1985).

\section{Considerações Finais}

É de suma importância realizar a prevenção e o manuseio correto de substâncias potencialmente danosas a pele e as mucosas para evitar a ocorrência acidentes. O cirurgião dentista tem papel essencial quanto ao manejo correto de pacientes que sofreram queimaduras, o que inclui a realização de anamnese e exame físico detalhados para análise do contato e do tipo de 
Research, Society and Development, v. 10, n. 4, e58410414418, 2021

(CC BY 4.0) | ISSN 2525-3409 | DOI: http://dx.doi.org/10.33448/rsd-v10i4.14418

substancia e suas orientações conforme os sinais e sintomas do paciente. Além de que encaminhamento médico de urgência é de extrema importância caso seja observado ingestão da substância no exame clinico.

\section{Referências}

Alfawaz, H. (2020). Chemical burn from direct application of aspirin onto a painful tooth. Saudi Endodontic Journal, $10(1), 65$.

Arevalo-Silva, C., Eliashar, R., Wohlgelernter, J., Elidan, J., \& Gross, M. (2006). Ingestion of caustic substances: a 15-year experience. Laryngoscope, 116(8), 1422-1426. doi:10.1097/01.mlg.0000225376.83670.4d

Bahnof, R. (2000). Intra-oral burns: rehabilitation of severe restriction of mouth opening: case report. Physiotherapy, 86(5), 263-266.

Baruchin, A. M., Lustig, J. P., Nahlieli, O., \& Neder, A. (1991). Burns of the oral mucosa: Report of 6 cases. Journal of Cranio-Maxillofacial Surgery, 19(2), 94-96. doi:https://doi.org/10.1016/S1010-5182(05)80615-8

Clayton, N. A., Ward, E. C., \& Maitz, P. K. (2015). Full thickness facial burns: Outcomes following orofacial rehabilitation. Burns, 41 (7), 1599-1606. doi:10.1016/j.burns.2015.04.003

Cowan, D., Ho, B., Sykes, K. J., \& Wei, J. L. (2013). Pediatric oral burns: a ten-year review of patient characteristics, etiologies and treatment outcomes. International journal of pediatric otorhinolaryngology, 77(8), 1325-1328.

Edlich, R., Farinholt, H.-M. A., Winters, K. L., Britt, L., \& Long III, W. B. (2005). Modern concepts of treatment and prevention of electrical burns. Journal of long-term effects of medical implants, 15(5).

Evans, E. W. (2017). Treating Scars on the Oral Mucosa. Facial Plast Surg Clin North Am, 25(1), 89-97. 10.1016/j.fsc.2016.08.008

Hagiwara, Y., Seki, K., \& Takahashi, Y. (2020). Oral chemical burn due to accidental ingestion of calcium oxide food desiccant in a patient with dementia. $J$ Int Med Res, 48(4), 300060520920065. doi:10.1177/0300060520920065

Haller Jr, J. A., Andrews, H. G., White, J. J., Tamer, M. A., \& Cleveland, W. W. (1971). Pathophysiology and management of acute corrosive burns of the esophagus: results of treatment in 285 children. Journal of pediatric surgery, 6(5), 578-584.

Kang, S., Kufta, K., Sollecito, T. P., \& Panchal, N. (2017). A treatment algorithm for the management of intraoral burns: A narrative review. Burns. doi:10.1016/j.burns.2017.09.006

Kurowski, J. A., \& Kay, M. (2017). Caustic Ingestions and Foreign Bodies Ingestions in Pediatric Patients. Pediatr Clin North Am, 64(3), 507-524. doi:10.1016/j.pcl.2017.01.004

Lopes, M. S., Cavalcante, I. d. S., Correia, R. S., Mendes, J. P. S., Barboza, D. L. L., Pereira, S. A., \& Beltrão, R. P. O. L. (2020). Development of sepsis in burn patients, a literature review. Research, Society and Development, 9(11), e3279119901. doi:10.33448/rsd-v9i11.9901

Nehrlich, J., Klöcking, H. P., Hentschel, H., \& Lupp, A. (2017). Oral Chemical Burns Reported to the Poisons Information Centre in Erfurt, Germany, from 1997 to 2014. J Burn Care Res, 38(6), e913-e922. doi:10.1097/bcr.0000000000000518

Nichter, L., Morgan, R., Bryant, C., Haines, P., Bacchetta, C., \& Edlich, R. (1985). Electric burns of the oral cavity. Comprehensive therapy, 11 (4), 65-71.

Rallan, M., Malhotra, G., Rallan, N. S., \& Mayall, S. (2013). Management of chemical burn in oral cavity. BMJ Case Rep, 2013. doi:10.1136/bcr-2013009083

Spranley, T. J., Winkler, M., Dagate, J., Oncale, D., \& Strother, E. (2012). Curing light burns. Gen Dent, 60(4), e210-214.

Sundararajan, D., Noonan, V., \& Gallagher, G. (2017). Oral Mucosal Chemical Burn. J Mass Dent Soc, 65(4), 31.

Swami, P. C., Raval, R., Kaur, M., \& Kaur, J. (2016). Accidental intraoral injection of formalin during extraction: case report. British Journal of Oral and Maxillofacial Surgery, 54(3), 351-352.

Vargo, R. J., Warner, B. M., Potluri, A., \& Prasad, J. L. (2017). Garlic burn of the oral mucosa: A case report and review of self-treatment chemical burns. The Journal of the American Dental Association, 148(10), 767-771.

Wason, S. (1985). The emergency management of caustic ingestions. The Journal of Emergency Medicine, 2(3), 175-182. 10.1016/0736-4679(85)90393-2 